\title{
Ultrasound Imaging Simulation and Echocardiographic Image Synthesis
}

\author{
Mingzhou Song, Robert M. Haralick, \\ University of Washington \\ Department of Electrical Engineering \\ Intelligent Systems Laboratory \\ Seattle, WA 98195, U.S.A. \\ \{msong,haralick\}@isl.ee.washington.edu
}

\author{
Florence H. Sheehan, \\ University of Washington \\ School of Medicine \\ Division of Cardiology \\ Seattle, WA 98195, U.S.A. \\ sheehan@u.washington.edu
}

\begin{abstract}
The paper presents a ray tracing ultrasound imaging simulation method that accounts for the effects of reflection, scattering and attenuation. Two-dimensional echocardiograhpic images were synthesized using this method. Nonlinear effects introduced by the signal processing units in an ultrasound imaging system were also emulated. Threedimensional triangular facet mesh models were employed in representing the heart structures and generating the synthetic two-dimensional echocardiographic images. The synthetic images agreed with the corresponding real ultrasound images in major ultrasound effects. No other prior work in echocardiographic image synthesis is known.
\end{abstract}

\section{Introduction}

Although ultrasound images have low signal-to-noise ratio, ultrasound imaging is especially suited for the beating heart, because the motion blur in ultrasound images is less severe than with other modalities due to its shorter image formation time.

A 3-D heart reconstruction method is being investigated by iteratively optimizing a 3-D heart mesh model using the discrepancy between the real ultrasound images and the synthetic heart images generated from the model being optimized. The ultrasound imaging simulation and the heart image synthesis part is reported in this paper. Ultrasound phenomena including reflection, scattering and attenuation were simulated. The simulation was done by the ray tracing rendering technique. The surface models of the heart structures used are developed by [4]. Nonlinear effects introduced by the signal processing units in an ultrasound imaging system were also emulated.

Simulation of ultrasound imaging has been reported in $[1,2]$. However, they either used no object model or simplified the material characteristics for different purposes.

\section{Physics of Ultrasound in the Heart}

\subsection{Reflection and Refraction}

The angle of incidence $\theta_{i}$ is defined as the angle between the surface normal to the interface and the direction of the incident sound wave. The angle of reflection $\theta_{r}$ is defined as the angle between the surface normal to the interface and the direction of the reflected sound wave. The angle of transmission $\theta_{t}$ is defined as the angle between the surface normal to the interface and the direction of the transmitted sound wave. All the angles must be between $0^{\circ}$ and $90^{\circ}$.

The reflected intensity of a plane compressional sound wave at specular boundary is given by

$$
I_{r}=I_{i}\left(\frac{Z_{2} \cos \theta_{i}-Z_{1} \cos \theta_{t}}{Z_{2} \cos \theta_{i}+Z_{1} \cos \theta_{t}}\right)^{2}
$$

where $I_{r}\left(W / m^{2}\right)$ is the reflected intensity, $I_{i}\left(W / m^{2}\right)$ is the incident intensity, $Z_{1}\left(\mathrm{~kg} / \mathrm{m}^{2} \mathrm{~s}\right)$ is the characteristic impedance of medium $1, Z_{2}\left(\mathrm{~kg} / \mathrm{m}^{2} \mathrm{~s}\right)$ is the characteristic impedance of medium 2 . The transmitted sound intensity is

$$
I_{t}=I_{i} \frac{4 Z_{2} Z_{1} \cos ^{2} \theta_{i}}{\left(Z_{2} \cos \theta_{i}+Z_{1} \cos \theta_{t}\right)^{2}}
$$

The relationships among $\theta_{i}, \theta_{r}$ and $\theta_{t}$ are $\theta_{r}=\theta_{i}$ and $\frac{\sin \theta_{t}}{c_{2}}=\frac{\sin \theta_{i}}{c_{1}}$, where $c_{1}$ is the velocity of sound in medium $1, c_{2}$ is the velocity of sound in medium 2 .

In non-specular reflection, the reflected waves go to many directions in addition to the direction of the perfect reflection. The reflected intensity to the incident direction is calculated as [7] $I_{r}=I_{i} W\left(\theta_{i}\right) \cos ^{n} \theta_{i}$, where $I_{r}$ is the reflected intensity to the direction of the incident light, $W\left(\theta_{i}\right)$ is the specular-refection coefficient of the interface and $n$ is the specular-refection exponent of the interface. 


\subsection{Attenuation as Scattering and Absorption}

Attenuation of the incident intensity $I_{i}$ is given by

$$
I_{l}=I_{i} \exp (-2 \alpha l)
$$

where $I_{l}$ is the attenuated intensity, $\alpha$ is the attenuation coefficient $\left(\mathrm{Np} \cdot \mathrm{cm}^{-1}\right.$ or $\left.\mathrm{cm}^{-1}\right)$ and $l$ is the distance between where the attenuation starts and the point to be observed.

The backscattered intensity $I_{s}\left(180^{\circ}\right)\left(W_{c m}^{-2} S r^{-1}\right)$ is the intensity of the scattered sound wave that goes backward in the opposite direction of the incident sound wave. The formula to calculate $I_{s}\left(180^{\circ}\right)$ is [5]

$$
I_{s}\left(180^{\circ}\right)=\frac{\eta_{b} V I_{i}}{l^{2}}=\frac{\sigma_{b} I_{i}}{l^{2}}
$$

where $\eta_{b}$ is the backscattering coefficient $\left(\mathrm{cm}^{-1} \mathrm{Sr}^{-1}\right), l$ is the radial distance between the scattering particle and the point to be observed, $\sigma_{b}$ is the differential backscattering cross section $\left(\mathrm{cm}^{2} \mathrm{Sr}^{-1}\right), V$ is unit volume $\left(\mathrm{cm}^{3}\right)$.

The differential scattering cross section $\sigma_{d}(\theta)$ of a single particle is given by [3]

$$
\sigma_{d}(\theta) \approx \frac{k^{4} a^{6}}{9}\left[\frac{G_{e}-G}{G}+\frac{3\left(\rho_{e}-\rho\right)}{2 \rho_{e}+\rho} \cos \theta\right]^{2}
$$

where $k$ is the wave number, $a$ is the radius of the particle, $G$ and $\rho$ are respectively, the compressibility $\left(\mathrm{cm}^{2} /\right.$ dyne $)$ and the mass density $\left(\mathrm{g} / \mathrm{cm}^{3}\right)$ of the fluid, $G_{e}$ and $\rho_{e}$ are, respectively, the compressibility and the mass density of the particle, $\theta$ is the angle between the direction of the incident ultrasound wave and the direction of the scattered wave, which is $180^{\circ}$ for backscattering.

The backscattering coefficient $\eta_{b}$ in blood is given by [8]

$$
\eta_{b}=\frac{\sigma_{b} W_{0}\left(1-W_{0}\right)^{4}}{V\left(1+2 W_{0}\right)^{2}}
$$

where $W_{0}$ is the hematocrit expressed as a fraction, $V$ is the volume of a single scattering particle, and $\sigma_{b}$ is the backscattering cross section of a single scattering particle.

The backscattering coefficient $\eta_{b}$ of tissue, such as fat and myocardium, is given by [6]

$$
\begin{aligned}
\eta_{b}=\frac{3 \bar{\mu}^{2} k}{4 \pi} & {\left[\tan ^{-1}\left(k b_{m}\right)+\frac{1}{9} \tan ^{-1}\left(3 k b_{m}\right)\right.} \\
& \left.-\frac{k b_{m}}{1+k^{2} b_{m}^{2}}-\frac{k b_{m}}{3\left(1+9 k^{2} b_{m}^{2}\right)}\right]
\end{aligned}
$$

where $\bar{\mu}$ is the root mean square velocity variation $\sqrt{\frac{(\Delta c)^{2}}{c^{2}}}$ ( $\Delta c$ is the time average change in the velocity as the ultrasound propagates through a region of tissue.), $b_{m}$ is the maximal correlation length which is an expression of the spatial distance over which the acoustic properties of a region remain correlated (based on autocorrelation) with some initial starting point in the tissue.
Figure 1. A mesh model for the left ventricle surface.

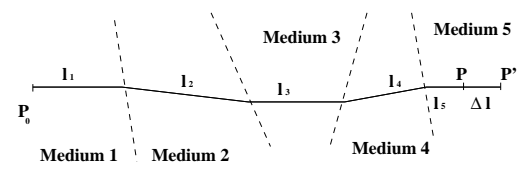

Figure 2. The ray tracing path.

\section{Heart Anatomy and Modeling}

The inner space of the heart is divided among four chambers. The left ventricle (LV) and the right ventricle (RV) are two chambers occupying the lower part of the heart at the places suggested by their names; the left atrium (LA) and the right atrium (RA) are the other two chambers that occupy the upper part of the heart at the places suggested by their names. The inner surface of the chambers is called the endocardium and the outer surface called the epicardium.

The piecewise smooth subdivision surface developed in [4] is used in 3-D representations of the heart surfaces. An example heart surface model is shown in Figure 1.

\section{Ultrasound Phenomenon Modeling by Ray Tracing}

The ultrasound beams are modeled as ideal rays. Each ray is traced. Along each ray, the intensity changed by attenuation and reflection is calculated. The intensity arriving at each location on the ray is either reflected or backscattered to the transducer.

\subsection{Ray Tracing Mechanism}

Figure 2 shows the ray tracing path. The wave starts at $\mathbf{P}_{0}$ with initial intensity $I_{S}$. Suppose the wave has traveled through $M$ media from $\mathbf{P}_{0}$. We use $\alpha^{(m)}$ to represent the attenuation coefficient of the $m$-th medium along the ray path, $\eta_{b}^{(m)}$ for the backscattering coefficient of medium $m, \theta_{i}^{(m)}$ and $\theta_{t}^{(m)}$ for the angles of incidence and transmis- 
sion from medium $m$ to medium $m+1, \alpha_{r}^{(m, m+1)}\left(\theta_{i}^{(m)}\right)$ and $\alpha_{t}^{(m, m+1)}\left(\theta_{i}^{(m)}\right)$ for the intensity reflection and transmission coefficients from medium $m$ to medium $m+1, l_{m}$ for the distance the wave has propagated through medium $m$. Hence there is a total of $M$ attenuations and $(M-1)$ refractions before the wave reaches $\mathbf{P}$. By the attenuation equation 3 and the refraction equation 2, it follows that

$I(\mathbf{P})=I_{S} \exp \left(-2 \sum_{m=1}^{M} \alpha^{(m)} l_{m}\right) \prod_{m=1}^{M-1} \alpha_{t}^{(m, m+1)}\left(\theta_{i}^{(m)}\right)$

At $\mathbf{P}$, the sound wave is backscattered if $\mathbf{P}$ is not on an interface, otherwise it is reflected.

In principle, there are many possible paths along which the back-propagated waves can arrive at $\mathbf{P}_{0}$. However, the probability of rays with large intensity getting to the transducer not via the reverse forward path is small. So we neglect the rays that get back to the transducer via paths other than the reverse forward path. Hence the angle of incidence from medium $m+1$ to medium $m$ is $\theta_{t}^{(m)}$ and the angle of transmission $\theta_{i}^{(m)}$.

When $\mathbf{P}$ is not on an interface, $I(\mathbf{P})$ is backscattered and the backscattered wave is still subject to attenuation and reflection. By the backscatter equation 4, the attenuation equation 3 and the refraction equation 2, the intensity $I_{R}(\mathbf{P})$ received at $\mathbf{P}_{0}$ is

$$
\begin{aligned}
I_{R}(\mathbf{P})= & I_{S} \frac{\eta_{b}^{(M)} V}{\left(\sum_{m=1}^{M} l_{m}\right)^{2}} \exp \left(-4 \sum_{m=1}^{M} \alpha^{(m)} l_{m}\right) \\
& \cdot \prod_{m=1}^{M-1} \alpha_{t}^{(m, m+1)}\left(\theta_{i}^{(m)}\right) \alpha_{t}^{(m+1, m)}\left(\theta_{t}^{(m)}\right)
\end{aligned}
$$

In Figure $2, \mathbf{P}^{\prime}$ is $\Delta l$ apart from $\mathbf{P}$ on the same ray path. For $\mathbf{P}^{\prime}$, the ray travels $\Delta l$ more in both forward and backward directions, which involves only one more attenuation and possibly one more reflection, the intensity for $\mathbf{P}$ can be utilized. So, based on the intensity $I_{R}(\mathbf{P})$, the intensity $I_{R}\left(\mathbf{P}^{\prime}\right)$ received by the transducer is

$$
I_{R}\left(\mathbf{P}^{\prime}\right)=\frac{l^{2}(\mathbf{P})}{(l(\mathbf{P})+\Delta l)^{2}} \exp \left(-4 \alpha^{(M)} \Delta l\right) I_{R}(\mathbf{P})
$$

where $l(\mathbf{P})=\sum_{m=1}^{M} l_{m}$.

The above recursive equation is for $\mathbf{P}^{\prime}$ and $\mathbf{P}$ in the same medium and not on any interface. It is easy to find the recursive equations for other situations similarly.

The recursive approach can save a large proportion of multiplications when $M$ is large.

\subsection{Ray Tracing Acceleration}

The ray tracing time depends on the total number of triangular facets making up all the structures, the total number of ray shots from the transducer and the time needed for each ray-facet intersection calculation.

Facet Plane Parameter Precalculation. The plane equation parameters for each triangular facet and the normals to each plane are precomputed since each facet plane may intersect with multiple rays.

Facet Candidate Selection. The triangular facets which do not intersect the imaging plane will not be involved in the simulation if the refraction is small enough to neglect. Hence, only those facets intersecting the imaging plane are selected as candidates for the ray-facet intersection.

Ray-Facet Intersection. It is time-consuming to find rayfacet intersections. When the intersection $\mathbf{P}$ between a ray and the plane of a facet is found, $\mathbf{P}$ and the facet are projected to a plane passing two coordinate axes. The inside/outside relationship between $\mathbf{P}$ and the facet is done in 2-D by looking at the inside/outside relationship between their projections.

Forward Ray Tracing Only. The ray has been assumed to go back to the transducer along the same path as it leaves the transducer. At each location along the path, attenuation or reflection happens twice: once in the ray's forward trip and once in the ray's backward trip. Since attenuation or reflection changes the intensity by a linear constant independent of the incident intensity, we can do pre-attenuation or pre-reflection for the backward trip in addition to the attenuation or reflection calculation in the forward trip. Hence, the backward ray tracing can be saved.

\section{Ultrasound Imaging System Nonlinear Ef- fects Simulation}

The most obvious nonlinear effects are introduced by the variable gain amplifier, the log compressor/demodulator and non-zero beam width in an ultrasound imaging system.

Signal loss from attenuation increases with distance from the transducer. A time controlled gain function (TGC) is applied to compensate for this. The TGC function is set manually by the sonographer each time before the scanning and can be emulated by a look-up table indexed by the distance to the transducer. However, since the TGC setting varies from case to case and is not available to the simulation algorithm, an approximation method called perfect $T G C$ is used. The perfect TGC compensates all attenuation perfectly as if no attenuation had happened.

Logarithm compression is used to reduce the dynamic range of the signal for display and was implemented in the simulation algorithm. 

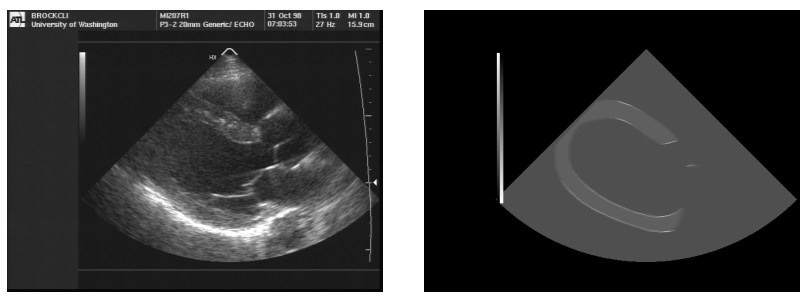

Figure 3. The real image (left) and the synthetic image (right).

To compensate for the error created by the assumption that the ultrasound source generates a planar wave with a zero-area wavefront in the ideal ray tracer, the blurring effects due to the beam width are modeled by doing an arithmetic average filtering on each point.

\section{Synthetic Images and Real Images}

Controlled experiments were done by generating synthetic images for several given imaging planes from the reconstructed 3-D heart mesh models of a series of patient studies. The corresponding real ultrasound images are available for comparison.

To compensate for the error in the estimation of tissue characteristics and empirical formulae, we applied piecewise linear regression on the grayscales of the synthetic images.

Figure 3 shows a synthetic image produced together with its corresponding real image.

The synthetic images look different from the real images in several ways. The differences are explained as follows:

- There are speckle noises in the real images whereas not in the synthetic images. The speckles are caused by the randomness of the scatterers, for example, blood cells, and the coherent interference of the reflected ultrasound waves. The random speckle can be simulated but it will not increase accuracy even though it makes the image more realistic-looking. In our project, realistic-appearance does not take the first priority.

- There are fewer structures in the synthetic images. This is because only the left ventricle was used in the simulation. Models of other structures are either being developed or neglected.

- The areas near the transducer look quite different. In clinical practice, the near field part in the image is not considered because the region has mixed unanticipated tissues.

Despite the above differences, the synthetic images agree with the real images at the endocardium, the wall and the epicardium of the left ventricle. The highlighted boundaries due to reflection at interfaces are caught in the synthetic images. These areas are of the most importance in 3-D heart reconstruction.

A group of medical ultrasound imaging experts assessed the synthetic images and endorsed their usage in the 3-D heart reconstruction project, for the major ultrasound effects are present and accurate in the synthetic images.

\section{Conclusions}

An ultrasound imaging simulation method using ray tracing technique has been described in this paper. The ultrasound phenomena including reflection, scattering and attenuation are simulated, which provides a way to generate synthetic images. The synthetic images can be used to evaluate the 3-D object model.

\section{References}

[1] J. D'hooge. Simulation of the scattering of ultrasound by tissues. Technical report, Katholieke Universiteit Leuven, Leuven, Belgium, May 1996.

[2] M. Garton. UT-Sim. Center for Nondestructive Evaluation, Iowa State University, Ames, Iowa, USA. See web page http://www.cnde.iastate.edu/staff/mgarton/mgarton.html.

[3] A. Ishimaru. Wave Propagation and Scattering in Random Media, volume 1 Single Scattering and Transport Theory. Academic Press, New York, USA, 1978.

[4] M. E. Legget, D. F. Leotta, E. L. Bolson, J. A. McDonald, R. W. Martin, X.-N. Li, C. M. Otto, and F. H. Sheehan. System for quantitative three-dimensional echocardiography of the left ventricle based on a magnetic-field position and orientation sensing system. IEEE Transactions on Biomedical Engineering, 45(4):494-504, April 1998.

[5] R. W. Martin. The interaction of ultrasound with tissue, approaches to tissue characterization and measurement accuracy. In C. M. Otto and A. S. Pearlman, editors, Practice of Clinical Echocardiography, pages 113-129. W.B. Saunders Company, Philadelphia, Pennsylvania, USA, 1997.

[6] C. M. Sehgal and J. F. Greenleaf. Scattering of ultrasound by tissues. Ultrasonic Imaging, 6:60-80, 1984.

[7] J. Warnock. A hidden-surface algorithm for computer generated half-tone pictures. Technical Report TR 4-15, NTIS AD-753 671, Computer Science Department, University of Utah, Salt Lake City, Utah, USA, June 1969.

[8] Y. W. Yuan and K. K. Shung. Ultrasonic backscatter from flowing whole blood: I. dependence on shear rate and hematocrit. Journal of the Acoustical Society of America, 84(1):52$58,1988$. 